\title{
Dose calculation of different eye substructures using a realistic eye model when treating ocular tumors with electron therapy
}

\author{
A. Vejdani-Noghreiyan ${ }^{\star}$, A. Ebrahimi-Khankook, E. Rahmani and M. Sakhaee \\ Physics Department, Faculty of Sciences, University of Neyshabur, Neyshabur, Iran.
}

Received 29 July 2015 - Accepted 28 April 2016

\begin{abstract}
One of the most frequent types of intraocular melanoma in adults is choroidal uveal melanoma. The most commonly used forms of radiation therapy are ophthalmic plaque brachytherapy and charged particle external radiation therapy. In the absence of adequate facilities for brachytherapy and proton therapy, electron therapy would be an efficient radiation therapy technique for treating eye melanomas. In the present work, the Monte Carlo code MCNPX 2.6.0 was used to calculate the dose distribution to substructures of the eye in electron therapy of three common choroidal tumors. The simulations were performed for 6 electron beams with nominal energies between $5 \mathrm{MeV}$ and $10 \mathrm{MeV}$ and also for 10 incidence angles. To identify suitable treatment plans, the tumor-to-sensitive zone dose ratios were estimated. Moreover, the equivalent doses delivered to healthy substructures of the eye were also calculated. The results indicate that for the treatment of tumors located at the posterior part of the eye, an electron beam with energy of $10 \mathrm{MeV}$ and incident angle of $45^{\circ}$ relative to the eye axis provides the best tumor coverage while optimally sparing other eye substructures. In addition, for tumors positioned on the upper and lower parts of the vitreous, electron beams with energy of $10 \mathrm{MeV}$, an azimuthal angle of $270^{\circ}$, and polar incident angles of $90^{\circ}$ and $105^{\circ}$, respectively, lead to appropriate dose delivery.
\end{abstract}

Keywords: eye melanoma / realistic eye model / electron beam / Monte Carlo simulation

\section{Introduction}

Radiation therapy is the most common technique for the treatment of ocular melanoma. Ocular treatment is aimed at conserving the eye with as much useful vision as possible (Stannard et al., 2013). Therefore, providing a safe and optimized treatment plan with less destructive effects on the sensitive part of the eye is essential. The most common technique for treating eye melanoma is brachytherapy. Brachytherapy is the placing of a radioactive source close to or into a tumor. However, in cases where the tumor is placed near the optic nerve, brachytherapy is less often used because of concerns about damage to the optic nerve (Höcht et al., 2005; Weber et al., 2005). The other technique used for treating ocular melanoma is charged-particle (external beam) radiation therapy. Radiation therapy with charged particles can potentially deliver maximum doses while minimizing irradiation of surrounding tissue, and it may be an effective form of radiation therapy (Terasawa et al., 2009). Currently, heavy charged particles such as protons and heavy ions are used to treat ocular melanoma in many radiation therapy centers. However, in radiation therapy centers in which adequate facilities for brachytherapy and proton therapy do not exist, electron or photon beam therapy is also used for treating ocular melanoma

\footnotetext{
^ vejdani@neyshabur.ac.ir
}

(Inoue et al., 2014). Several authors have studied the performance of electron beam therapy for treating different types of eye melanomas (Al-Beteri and Raeside, 1992; Blach et al., 1996; Lee et al., 2005). Lee et al. (2005) compared the dose distribution and dose volume histograms (DVHs) for photon therapy, electron therapy, intensity-modulated radiation therapy and standard proton therapy. The tumor volumes from 3 patients with retinoblastoma were investigated. The results showed about $95 \%$ of the target volume was covered by the prescription dose using an electron field (Lee et al., 2005).

Therefore, in the lack of brachytherapy and proton therapy facilities, electron therapy can also be used effectively for cancer treatment because electrons deposit their energies in a short range of the distance.

In initial studies, the contraindications and complexities of radiation were determined through trial and error (Abramson et al., 1981). Today, the treatment plan is designed by planning software systems based on medical imaging. However, treatment planning algorithms have some serious limitations such as unrealistic prediction of hot and cold spots in inhomogeneous phantoms and significant deviations between the results of calculations with the algorithms and measurements, which are up to $40 \%$ for areas located outside the radiation beam (IAEA, 2004; Ding et al., 2005). In addition, a complex threedimensional inhomogeneous phantom is composed of different 
materials and densities, whereas these algorithms even have limitations in predicting high- and low-dose variations for simple three-dimensional inhomogeneities (Ding et al., 2005). Moreover, using a Monte Carlo-based system in patient dose estimation showed an excellent agreement between the calculated and measured dose (Ding et al., 2006; Mohammadi et al., 2015). Thus, using the combination of an anatomical model with Monte Carlo code would provide acceptable results.

For many years, in dosimetric calculations, the eye was designed with a simple spherical model (Kramer et al., 1982). In 1938, Goldman and Liechti indicated that radiation can affect the eye lens considerably in tropical regions (Goldman and Liechti, 1938). Based on clinical and experimental data, Charles and Brown determined the averaged dimensions of different parts of the eye for the adult population about 40 years later (Charles and Brown, 1975). According to the literature, radiation can directly injure the cornea, conjunctiva, iris and retina at doses greater than 30 Gy (Halperin et al., 2013). Aqueous and vitreous humors are similar to water in many respects and any change to these structures may damage the cornea, lens or retina (Voke, 1999). The lens is the most radiosensitive structure in the eye and thus delivering doses between 2.5 and 6.5 Gy to the lens is associated with developing lens injury, which is called a cataract (Halperin et al., 2013). Nevertheless, recent reports state that the threshold dose of the sensitive region of the lens for cataract induction, if any, is much lower than 0.5 Gy (Behrens et al., 2009).

Because the sensitivities of various parts of the eye are not similar, it is very important to accurately simulate substructures of the eye and to determine their exact locations and doses (Charles and Brown, 1975). Worgul and Merriam also specified different types of cell populations in the lens and concluded that the epithelial cells in the germinative zone (GZ) of the lens are the most radiosensitive cells (Worgul and Merriam, 1980). Recently, different scientific groups developed realistic mathematical eye models based on data reported by Charles and Brown, and Worgul and Merriam (Behrens et al., 2009; Nogueira et al., 2011). In these models, developed for dosimetry purposes, different substructures of the eye were distinguished.

In the present study, the Monte Carlo MCNPX code was used to calculate the dose distribution to the substructures of the eye following external beam electron radiation therapy of a choroidal uveal melanoma. For this purpose, a realistic eye model developed by Worgul et al. and implemented by Nogueira et al. was used and schematic dome-shaped intraocular melanomas were simulated at three different positions of the choroid and irradiated by clinical electron beams. The specifications of the simulated tumors were taken from Lesperance et al. (2014). In order to consider the effect of head-scattered radiation on the dose to the eye substructures, Nogueira et al.'s model was inserted into the whole-body UF-ORNL phantom (Han et al., 2006).

Simulations were performed for 6 electron beams with different nominal energies between 5 and $10 \mathrm{MeV}$ and for 10 different incidence angles. To optimize the treatment plan for a patient, one of the main issues is maximizing the dose delivered to the tumor tissue while minimizing doses received by healthy structures. In clinical practice, suitable radiation

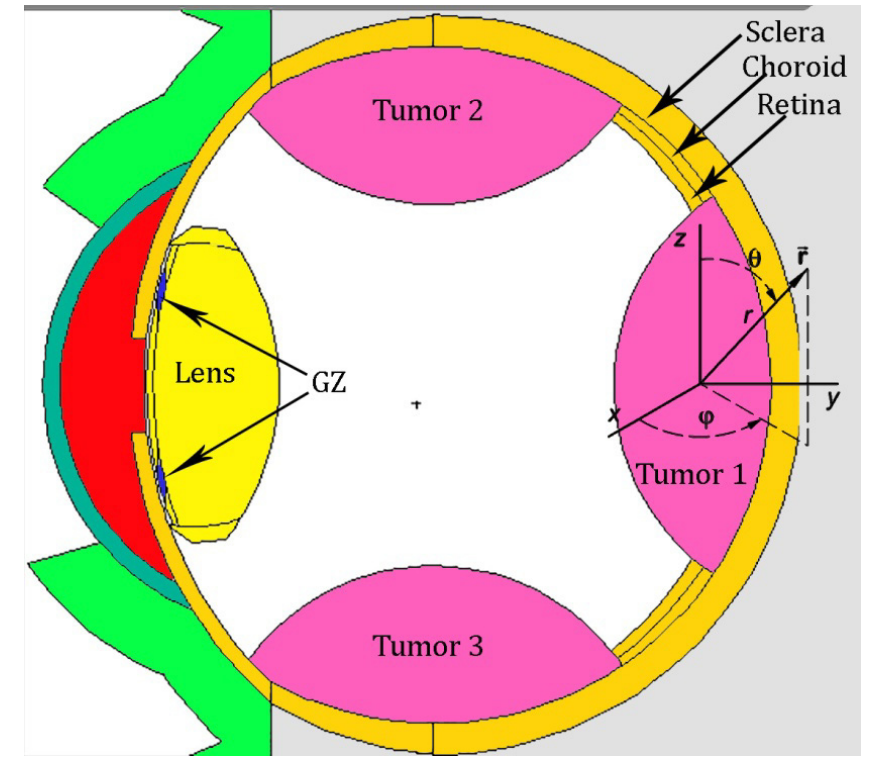

Figure 1. Choroidal melanoma is located in the left eye of the phantom. All types of tumors are specified.

incident angles (RIAs) are chosen based on the tumor coverage, dose uniformity and tumor-to-sensitive zone dose ratio $\left(D_{\text {Tumor }} / D_{\mathrm{GZ}}\right)$. The necessary dose to destroy the tumor was estimated from the clinical study by Muller et al. (2005), who performed a comprehensive study on 102 patients with uveal melanoma treated with fractionated stereotactic radiation therapy between 1999 and 2007 (Muller et al., 2005). The choroidal melanoma investigated in this study can be included in this category. Accordingly, a prescription dose of $50 \mathrm{~Gy}$ is considered enough to destroy tumor cells (Muller et al., 2005).

\section{Material and methods}

\subsection{Geometry of the eye model}

Nogueira et al. developed a realistic eye model based on the details provided in Worgul and Merriam 's report (Worgul and Merriam, 1980; Nogueira et al., 2011). In this study, the dimensions and geometries of the eye substructures were taken from this model. Nogueira et al.'s eye model includes the eye substructures the eyelid, cornea, anterior chamber, lens, sclera, vitreous humor, retina and choroid (Figure 1). In this model, the anterior portion of the eye lens, named the lens epithelium, is divided into four zones: the central zone, germinative zone (GZ), transitional zone and meridional rows. The GZ is the primary site of the mitotic activity and thus is the most radiosensitive region of the eye lens (Sakhaee et al., 2015).

\subsection{Tumor location inside the eye}

Choroidal uveal melanoma is a common intraocular melanoma usually observed in adults. This type of melanoma is frequently dome-shaped and is modeled as a segment of a 
Table 1. Specifications of electron beam sources (Righi et al., 2013).

\begin{tabular}{lccccc}
\hline \multirow{3}{*}{ Novac7 } & $\begin{array}{c}\text { Nominal Energy } \\
(\mathbf{M e V})\end{array}$ & $\begin{array}{c}\boldsymbol{E}_{\text {peak }} \\
(\mathbf{M e V})\end{array}$ & $\begin{array}{c}\boldsymbol{E}_{\text {average }} \\
(\mathbf{M e V})\end{array}$ & $\begin{array}{c}\boldsymbol{E}_{\max } \\
(\mathbf{M e V})\end{array}$ & $\begin{array}{c}\text { FWHM } \\
(\text { degree })\end{array}$ \\
& 5 & 5.9 & 4.7 & 6.8 & 3.5 \\
& 7 & 7.1 & 5.9 & 8.4 & 3.1 \\
LIAC & 9 & 8.2 & 6.8 & 9.7 & 2.8 \\
& 6 & 5.8 & 5.5 & 8.0 & 3.5 \\
& 10 & 8.4 & 6.8 & 10 & 3.1 \\
\hline
\end{tabular}

Note: $E_{\text {peak }}$ is the most probable energy, $E_{\text {average }}$ is the mean energy and $E_{\max }$ is the maximum energy.

sphere (Lesperance et al., 2014). In this work, three mediumsized tumors were simulated based on the tumor model presented by Lesperance et al. (2014). Tumor 1, which was located in the posterior side of the choroidal region, was entirely consistent with Lesperance et al.'s (2014) tumor model. The tumor diameters in the $\mathrm{z}$ and $\mathrm{y}$ directions were $12.014 \mathrm{~mm}$ and $5 \mathrm{~mm}$, relatively. Although choroidal melanoma commonly occurs in the posterior side of the choroid, it may also form in other orientations (Lesperance et al., 2014). Therefore, two other tumor positions were modeled at the medial side of the eye, one on the upper part (tumor 2) and another on the lower part (tumor 3 ) centered along the $\mathrm{z}$-axis. The same shape as tumor 1 was considered for all three tumor geometries (see Figure 1).

\subsection{Monte Carlo simulation}

In this study, MCNPX code version 2.6.0 was used to simulate the ocular treatment and model particle transport (Pelowitz, 2008). Based on Novac7 and LIAC linear accelerators (linacs), electron beams with an irradiation field of $5 \times 5 \mathrm{~cm}^{2}$ were considered as radiation sources (Righi et al., 2013). The specifications of the simulated sources are presented in detail in Table 1. Since the lens is the most sensitive structure, it should be shielded from irradiation to prevent the formation of cataracts (Rykers et al., 2003). In this work, in order to reduce the destructive effects of the radiation on healthy tissues, an appropriate shield was placed in the path of the radiation beam. The shield had a hole that was substantially aligned with the tumor area and provided a 1-mm margin around the target volume. This shield is made of Cerrobend, which is an alloy consisting of bismuth (50\%), lead (26.7\%), tin $(13.3 \%)$ and cadmium (10\%), with a melting temperature of approximately $70{ }^{\circ} \mathrm{C}$ and a density of about $9.4 \mathrm{~g} \mathrm{~cm}^{-3}$ at $20^{\circ} \mathrm{C}$ (Di Venanzio et al., 2015). Using Cerrobend blocks is common in radiation therapy because of its flexibility, also allowing the dose delivery by a more complex specific beam (Taherkhani et al., 2010; Farajollahi et al., 2015). The dosimetric properties of small-field electron beams shaped by Cerrobend blocks were previously investigated by Di Venanzio et al. (2015).

As mentioned before, in order to estimate the dose received by the sensitive organs in the head region, the eye model was inserted into the mathematical UF-ORNL whole-body phantom (Han et al., 2006). For each tumor, optimized treatment plans with and without considering the dose delivered to the main organs in the head region were determined, and the doses received by the GZ were compared during the two selected treatment plans. For all eye substructures and important organs of the head region, absorbed doses were calculated using a collision heating tally $(+\mathrm{F} 6)$. Moreover, dose distribution profiles were also obtained over the eye volume using a rectangular mesh tally. The meshes were defined as cubes with a size of $0.5 \times 0.5 \times 0.5 \mathrm{~mm}^{3}$, and the deposited energy in each cube was assessed by tally type 3 . Since the mean absorbed dose does not give information regarding the dose uniformity, dose volume histograms (DVHs) within the tumor volume were obtained. A DVH is a plot of a cumulative dose volume frequency distribution which summarizes graphically the simulated radiation distribution within a volume of interest (Drzymala et al., 1991). Although it was not possible to establish a mesh tally block which would exactly encompass the tumor, a rectangular mesh block with a mesh size small enough was used to closely cover the tumor. In addition, a postprocessing FORTRAN code was developed to selectively tabulate the dose within the meshes included within the true tumor's geometrical boundaries.

In consultation with a physician, calculations were performed for electron beams with 6 energies ranging from $5 \mathrm{MeV}$ to $10 \mathrm{MeV}$, and for 10 different RIAs. The RIAs were considered relative to the iso-center of the tumor and were defined by the azimuthal angle (the angle relative to the $\mathrm{x}$-axis, $\varphi$ ) and the polar angle (the angle relative to the z-axis, $\theta$ ). The beam polar angles varied from $45^{\circ}$ to $135^{\circ}$ in $15^{\circ}$ increments at an azimuthal angle of $270^{\circ}$. In addition, the beam azimuthal angles varied from $285^{\circ}$ to $315^{\circ}$ in $15^{\circ}$ increments at a polar angle of $90^{\circ}$.

Considering the small size of the GZ region and mesh dimensions, for low-energy electrons $2 \times 10^{9}$ histories were transported. However, for higher-electron energies, a smaller number of histories was required to reach the acceptable uncertainties. Finally, in most cases, the equivalent doses of different parts of the eye and some sensitive organs in the head region such as the brain and sinuses reached a relative uncertainty below $1 \%$. The exception was the GZ, for which in the worst situations the relative uncertainty was about $3 \%$. In addition, all of the meshes belonging to the tumor volume had reasonable statistical uncertainties of around 3.5\%, except for the low electron energy, at which they approached $4 \%$. Calculations were run on a personal computer with a $3.50 \mathrm{GHz}$ Intel core i7 processor and 8 GB RAM, operated by Microsoft Windows 7. The average running time of each program was over 4 days. 
Table 2. The equivalent dose delivered to the GZ $\left(D_{\mathrm{GZ}}\right)$ in units Sv for all beam angles of incidence and all electron energies.

\begin{tabular}{|c|c|c|c|c|c|c|c|c|c|c|c|}
\hline & \multicolumn{11}{|c|}{ RIA } \\
\hline & $\varphi$ & & & & $270^{\circ}$ & & & & $285^{\circ}$ & $300^{\circ}$ & $315^{\circ}$ \\
\hline & $\theta$ & $45^{\circ}$ & $60^{\circ}$ & $75^{\circ}$ & $90^{\circ}$ & $105^{\circ}$ & $120^{\circ}$ & $135^{\circ}$ & & $90^{\circ}$ & \\
\hline \multirow{6}{*}{ Tumor 1} & 5 & 400.3 & 17.82 & 97.3 & 166.8 & 98.47 & 17.3 & 383.8 & 153.7 & 85.02 & 2.511 \\
\hline & 6 & 150 & 11.9 & 82.32 & 143.5 & 82.02 & 11.41 & 147.8 & 131.2 & 71.89 & 1.754 \\
\hline & 7 & 116.8 & 8.949 & 72.7 & 128.2 & 73.07 & 8.751 & 107.7 & 117 & 63.56 & 1.379 \\
\hline & 8 & 50.38 & 6.403 & 65.77 & 116 & 65.95 & 6.376 & 54.46 & 106.1 & 57.57 & 1.101 \\
\hline & 9 & 60.94 & 6.316 & 63.82 & 113.2 & 64.36 & 5.818 & 60.72 & 103.1 & 56.5 & 0.9512 \\
\hline & 10 & 17.87 & 2.905 & 51.75 & 91.79 & 51.61 & 2.805 & 18.84 & 84.76 & 45.52 & 0.5798 \\
\hline \multirow{6}{*}{ Tumor 2} & 5 & 19.66 & 5.673 & 0.3023 & 0.2411 & 3.323 & 55.81 & 132.1 & 0.2848 & 0.3689 & 0.6301 \\
\hline & 6 & 17 & 4.586 & 0.221 & 0.192 & 2.957 & 51.75 & 119.2 & 0.2241 & 0.2741 & 0.4523 \\
\hline & 7 & 16.16 & 3.825 & 0.1903 & 0.1452 & 2.514 & 48.72 & 110.3 & 0.1742 & 0.2152 & 0.3509 \\
\hline & 8 & 11.81 & 2.697 & 0.1548 & 0.1371 & 2.4 & 46.22 & 101.6 & 0.1609 & 0.1994 & 0.2833 \\
\hline & 9 & 12.72 & 2.622 & 0.15 & 0.1161 & 2.233 & 45.44 & 100.6 & 0.1353 & 0.1695 & 0.2627 \\
\hline & 10 & 6.379 & 1.279 & 0.09186 & 0.08668 & 1.836 & 40.33 & 84.6 & 0.1039 & 0.1212 & 0.1657 \\
\hline \multirow{6}{*}{ Tumor 3} & 5 & 133 & 55.31 & 4.915 & 0.2635 & 0.3903 & 5.757 & 19.98 & 0.3068 & 0.3949 & 0.6193 \\
\hline & 6 & 119 & 51.14 & 4.42 & 0.2057 & 0.293 & 4.531 & 17.3 & 0.2466 & 0.2968 & 0.4442 \\
\hline & 7 & 109.8 & 48.1 & 3.998 & 0.253 & 0.2466 & 3.788 & 15.63 & 0.1739 & 0.2254 & 0.3532 \\
\hline & 8 & 100.5 & 45.34 & 3.875 & 0.1397 & 0.1889 & 2.666 & 12.13 & 0.1616 & 0.2061 & 0.2994 \\
\hline & 9 & 99.92 & 44.6 & 3.591 & 0.125 & 0.1869 & 2.854 & 12.71 & 0.1423 & 0.1735 & 0.2617 \\
\hline & 10 & 84.54 & 39.61 & 3.033 & 0.09206 & 0.1097 & 1.327 & 6.517 & 0.1092 & 0.131 & 0.1969 \\
\hline
\end{tabular}

\section{Results}

In order to study the probability of cataract induction, the equivalent dose delivered to the $\mathrm{GZ}\left(D_{\mathrm{GZ}}\right)$ for the three tumors investigated and all the different RIAs are tabulated in Table 2. All data reported in this work were normalized by $50 \mathrm{~Sv}$ at the tumor. As can be seen, for the first tumor, the value of $D_{\mathrm{GZ}}$ fluctuated between $0.57 \mathrm{~Sv}$ and $400.3 \mathrm{~Sv}$, while for the second and third tumors, this ratio varied narrowly up to about $130 \mathrm{~Sv}$. Moreover, it can be seen that for all treatment plans considered for tumor 1, the GZ receives a higher dose than the dose limit for cataract induction. Nevertheless, the minimum GZ dose occurs for the plan with polar and azimuthal angles of $90^{\circ}$ and $315^{\circ}$, respectively, and a $10 \mathrm{MeV}$ electron beam. For tumors 2 and 3 , most of the treatment plans lead to a GZ below the lens dose limit. However, the lower GZ doses during the treatment of tumors 2 and 3 are assessed with polar and azimuthal angles of $90^{\circ}$ and $270^{\circ}$, respectively, and $10 \mathrm{MeV}$ energy.

According to clinic-based studies, the aim of radiotherapy is to deliver a lethal dose to the tumor volume while sparing the adjacent radiosensitive healthy tissues (Swakon et al., 2010). Therefore, to determine the most appropriate treatment plans, five plans which led to minimum doses of the GZ were selected for each tumor.

In order to determine appropriate treatment plans for each tumor, the DVHs of tumor volumes and tumor coverage were also investigated. Figure 2 illustrates the DVHs for the best treatment plans for tumors 1, 2 and 3. These DVHs were obtained by the plans with polar and azimuthal angles of $90^{\circ}$ and $315^{\circ}$ and $10 \mathrm{MeV}$ electron exposure, polar and azimuthal angles of $90^{\circ}$ and $270^{\circ}$ and $10 \mathrm{MeV}$ energy, and polar and azimuthal angles of $105^{\circ}$ and $270^{\circ}$ and $10 \mathrm{MeV}$ electrons for treating tumors 1,2 and 3 , respectively. It can be seen that for tumors 1 and 2 the treatment plans provide the same uniformity; however, the uniformity of the dose in tumor 3 is slightly lower than that of the other tumors.

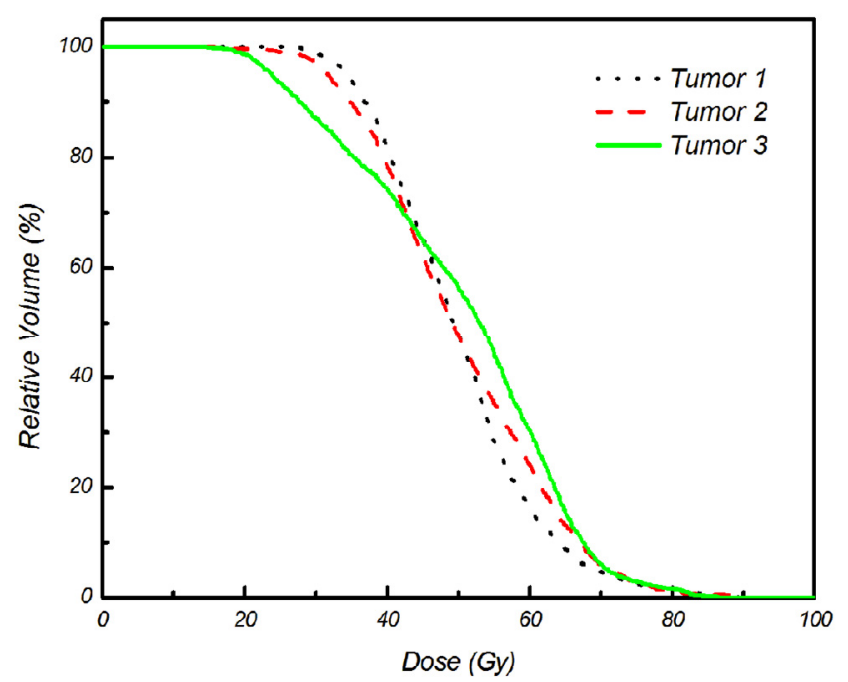

Figure 2. DVHs of tumor volume for the most appropriate treatment plans for different tumors.

Figures 3 to 5 indicate the dose profiles for the chosen plans. In these figures, the tumor volumes are distinguished by dashed lines. It is obvious that the tumor volume was fully covered by the irradiation field.

\section{Discussion}

This study is based on the need for using electron therapy techniques to treat ocular melanoma in the lack of brachytherapy and proton therapy facilities. Since the GZ in the eye lens is a radiosensitive tissue, formulation of treatment plans involving less damage to the GZ is a very significant issue.

Table 2 shows the equivalent dose of the GZ for the three tumors investigated. It can be seen that most of the irradiation 


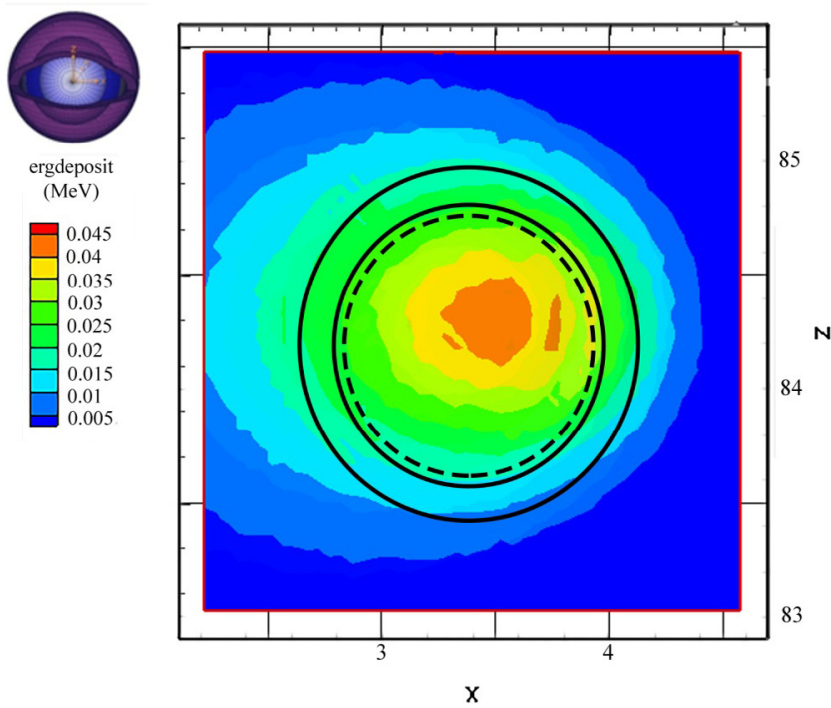

Figure 3. Energy deposition distribution in tumor 1: $\theta=90^{\circ}, \varphi=$ $315^{\circ}$ and $E=10 \mathrm{MeV}$.

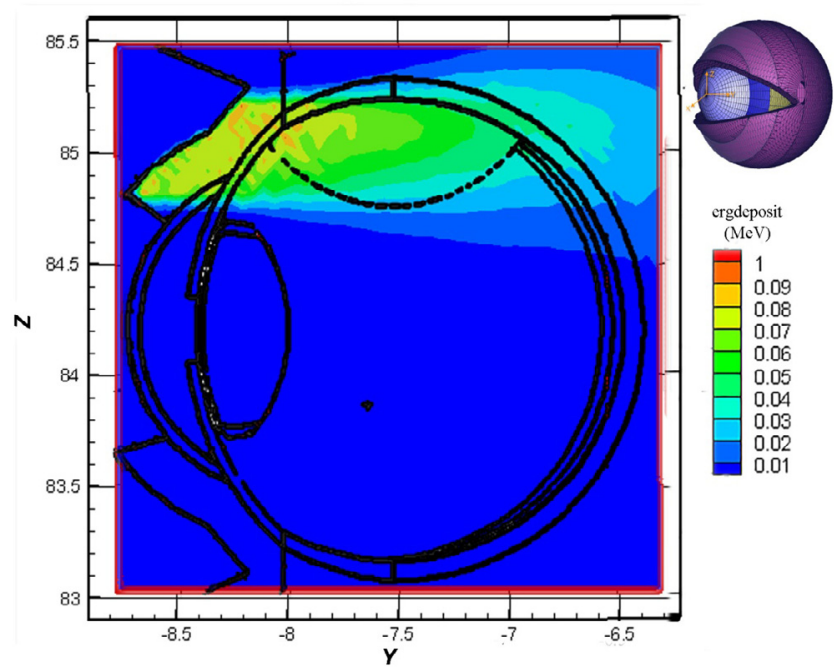

Figure 4. Energy deposition distribution in tumor 2: $\theta=90^{\circ}, \varphi=$ $270^{\circ}$ and $E=10 \mathrm{MeV}$.

exposures used for treating tumor 1 led to a high GZ dose. Tumor 1 is located on the posterior side of the eye at the back of the lens so that, for most treatment plans, the electron beam passes first through the GZ. Considering the relative position of tumor 1 and the sensitive zone and because of the existing symmetry, exposing an electron beam with energy of $10 \mathrm{MeV}$ along an edge of the cone with an opening angle of $45^{\circ}$ relative to the optic axis of the eye provides an appropriate dose distribution for tumor 1 .

Because of the absence of the eyelid in the eye corner, the edge of the cone was selected so that the electrons hit the eye directly. Table 2 shows the lowest GZ doses obtained by electron beams with an azimuthal angle of $315^{\circ}$. For these exposures, a small part of the GZ may be placed in the beam path and thus the GZ dose decreases.

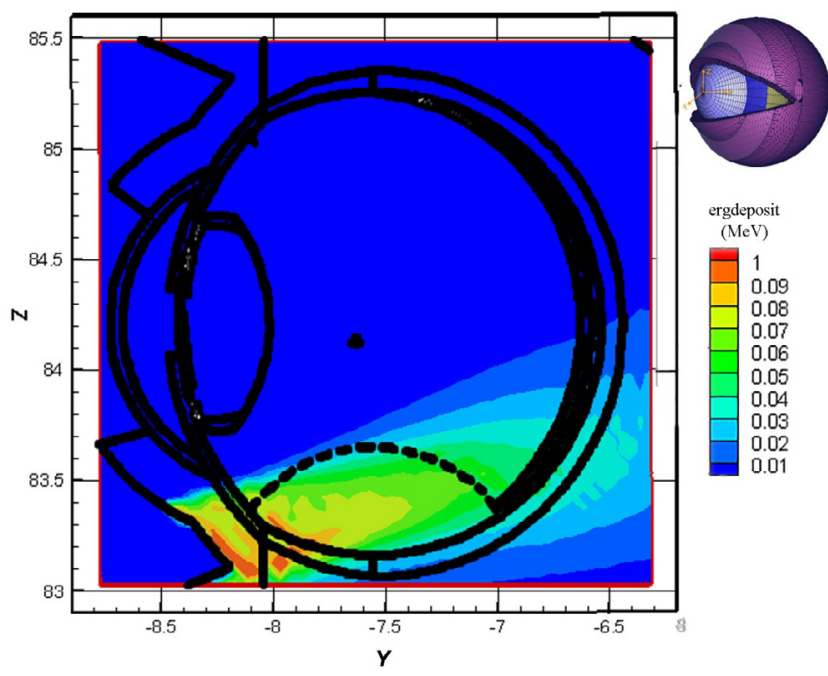

Figure 5. Energy deposition distribution in tumor 3: $\theta=105^{\circ}, \varphi=$ $270^{\circ}$ and $E=10 \mathrm{MeV}$.

For tumors 2 and 3, which are located on the top and bottom of the eye bulb, doses to the GZ are considerably lower than that of tumor 1 . The minimum GZ dose was obtained at $270^{\circ}$ azimuthal and $90^{\circ}$ polar angles for both of them.

To survey the probability of secondary cancer, the doses received by the other substructures of the eye and sensitive parts in the head region were also estimated. According to Table 3 for all exposure conditions, the equivalent doses of the eye substructures are less than their dose limit. Moreover, the doses delivered to the sensitive organs in the head region are very small.

To determine the appropriate treatment plan for each tumor, the DVHs and tumor coverage were also investigated. Among the five plans which received the minimum GZ dose, the plans that led to a more uniform dose distribution in tumor volume were chosen as the most appropriate treatment plans. The DVHs and dose profiles of the selected plans are shown in Figures 2 to 5 . In the case of tumors 1 and 2 the most appropriate plans are the same plans which provide the minimum GZ dose. However, the best exposure conditions for tumor 3 were obtained using a $10 \mathrm{MeV}$ electron beam with $105^{\circ}$ polar and $270^{\circ}$ azimuthal angles.

According to the results obtained, in the lack of brachytherapy and proton therapy facilities, electron therapy could be an acceptable and reliable method for treating ocular melanoma, although it may not always be the best technique. A simple comparison between the results reported in the present work and that of Lesperance et al. (2014) is shown in Table 4. The results indicate that the ratio of the total tumor dose to the lens dose is higher using electron therapy than brachytherapy. It is worth mentioning that in the electron therapy technique, the tumor region is exposed externally using a parallel beam. Moreover, the shield placed in the path of the beam protects the lens region against the radiation beam. Using brachytherapy, it is not possible to protect the lens and thus the lens may receive a high dose. However, the brachytherapy technique provides a more uniform dose distribution than electron therapy. However, when a brachytherapy facility is not avail- 


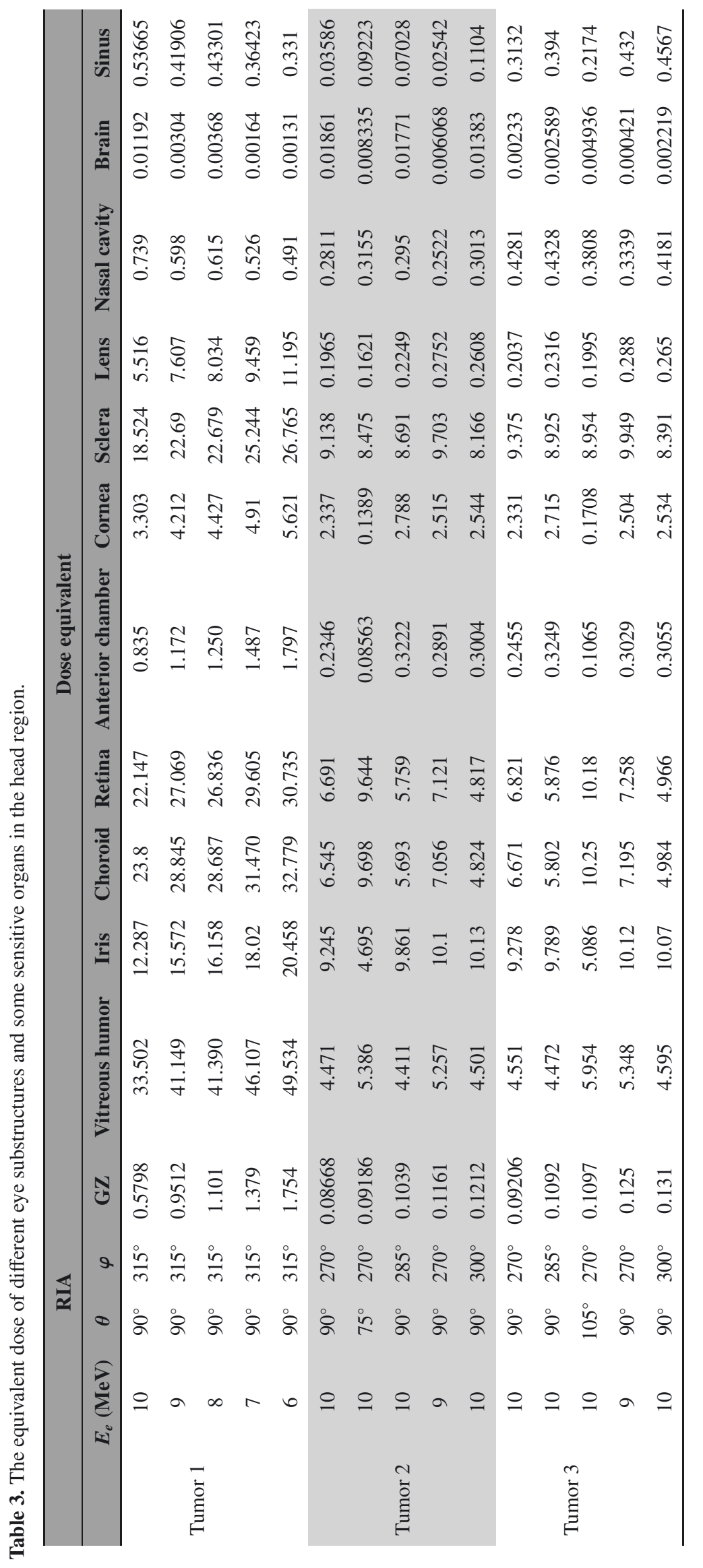


Table 4. Comparison of the ratio of $D_{\text {Tumor }} / D_{\text {Lens }}$ obtained by electron therapy (the present study) and brachytherapy (Lesperance et al., 2014) techniques.

\begin{tabular}{lcc}
\hline $\begin{array}{l}\text { Radiation Therapy } \\
\text { Technique }\end{array}$ & Type of exposure & $\boldsymbol{D}_{\text {Tumor }} / \boldsymbol{D}_{\text {Lens }}$ \\
\hline Brachytherapy & ${ }^{125} \mathrm{I}(\mathrm{COMS})$ & 10 \\
& ${ }^{103} \mathrm{Pd}(\mathrm{COMS})$ & 13 \\
Electron therapy & $10 \mathrm{MeV}$ electron beam & 9 \\
\hline
\end{tabular}

able or when the tumor is placed near the optic disc or lens region, electron therapy could be a choice for treating ocular melanoma.

\section{Conclusion}

The most realistic model of the eye was simulated to design an appropriate and safe treatment plan for treating intraocular melanoma. To achieve this aim, three types of choroidal uveal melanomas were considered and 60 irradiation conditions were investigated. It was found that successful treatment for eye tumors while simultaneously preserving the eye and healthy tissues can be achieved by oblique radiation of electron beams. The optimized RIAs and beam energies are as follows: tumor 1: $\theta=90^{\circ}, \varphi=315^{\circ}$ and $E=10 \mathrm{MeV}$; tumor 2: $\theta=90^{\circ}, \varphi=270^{\circ}$ and $E=10 \mathrm{MeV}$; and tumor $3: \theta=105^{\circ}$, $\varphi=270^{\circ}$ and $E=10 \mathrm{MeV}$.

\section{References}

Abramson D.H., Jereb B., Ellsworth R.M. (1981) External beam radiation for retinoblastoma, Bull. New York Acad. Med. 57, 787-803.

Al-Beteri A.A., Raeside D.E. (1992) Optimal electron-beam treatment planning for retinoblastoma using a new three-dimensional Monte Carlo-based treatment planning system, Med. Phys. 19, 125.

Behrens R., Dietze G., Zankl M. (2009) Dose conversion coefficients for electron exposure of the human eye lens, Phys. Med. Biol. 54, 4069-4087.

Blach L.E., McCormick B., Abramson D.H. (1996) External beam radiation therapy and retinoblastoma: long-term results in the comparison of two techniques, Int. J. Radiat. Oncol. 35, 45-51.

Charles M.W., Brown N. (1975) Dimensions of the human eye relevant to radiation protection, Phys. Med. Biol. 20, 202-218.

Ding G.X., Cygler J.E., Christine W.Y., Kalach N.I., Daskalov G. (2005) Comparison of electron beam dose calculation accuracy between treatment planning systems using either a pencil beam or a Monte Carlo algorithm, Int. J. Radiat. Oncol. Biol. Phys. 63 , 622-633.

Ding G.X., Duggan D.M., Coffey C.W., Shokrani P., Cygler J.E. (2006) First macro Monte Carlo based commercial dose calculation module for electron beam treatment planning-new issues for clinical consideration, Phys. Med. Biol. 51, 2781-2799.

Di Venanzio C., Marinelli M., Tonnetti A., Verona-Rinati G., Bagalà P., Falco M.D., Guerra A.S., Pimpinella M. (2015) Comparison between small radiation therapy electron beams collimated by Cerrobend and tubular applicators, J. Appl. Clin. Med. Phys. 16, 329-335.
Drzymala R.E., Mohan R., Brewster L., Chu J., Goitein M., Harms W., Urie M. (1991) Dose-volume histograms, Int. J. Radiat. Oncol. 21, 71-78.

Farajollahi A.R., Bouzarjomehri F., Kiani M. (2015) Comparison between Clinically Used Irregular Fields Shielded by Cerrobend and Standard Lead Blocks, J. Biomed. Phys. Eng. 5, 77.

Goldman H., Liechti A. (1938) Experimental investigation of the etiology of roentgen cataracts, Arch. F. Ophth. 138, 723.

Han E.Y., Bolch W.E., Eckerman K.F. (2006) Revisions to the ORNL series of adult and pediatric computational phantoms for use with the MIRD schema, Health Phys. 90, 337-356.

Halperin E.C., Waser D.E., Perez C.A., Brady L.W. (2013) Clinical radiation oncology. In: Perez $\mathcal{E}$ Brady's principles and practice of radiation oncology. pp. 626-1752. Lippincott Williams \& Wilkins. Philadelphia, New York, London.

Höcht S., Stark R., Seiler F., Heufelder J., Bechrakis N.E., Cordini D., Marnitz S., Kluge H., Foerster M.H., Hinkelbein W. (2005) Proton or Stereotactic Photon Irradiation for Posterior Uveal Melanoma?. Strahlenther Onkol. 181, 783-788.

IAEA (2004) Technical reports series No.430. Commissioning and quality assurance of computerized planning systems for radiation treatment of cancer. pp. 47-57.

Inoue T., Masai N., Ryoong-Jin O.H., Shiomi H., Hashida N. (2014) Adaptive replanning intensity-modulated radiotherapy for choroidal metastasis of breast cancer using optical coherence tomography, J. Radiat. Res. 55, 502-508.

Kramer R., Zankl M., Williams G., Drexler G. (1982) The calculation of dose from external photon exposures using reference human phantoms and Monte Carlo methods: Part I. The male (ADAM) and Female (EVA) adult mathematical phantoms. GSF-Report S885 .

Lee C.T., Bilton S.D., Famiglietti R.M., Riley B.A., Mahajan A., Chang E.L., Maor M.H., Woo S.Y., Cox J.D., Smith A.R. (2005) Treatment planning with protons for pediatric retinoblastoma, medulloblastoma, and pelvic sarcoma: how do protons compare with other conformal techniques?. Int. J. Radiat. Oncol. 63, 362372.

Lesperance M., Inglis-Whalen M., Thomson R.M. (2014) Modelbased dose calculations for COMS eye plaque brachytherapy using an anatomically realistic eye phantom, Med. Phys. 41, 021717 .

Mohammadi N., Miri-Hakimabad H., Rafat-Motavalli L., Akbari F., Abdollahi S. (2015) Patient-specific voxel phantom dosimetry during the prostate treatment with high-energy linac, $J$. Radioanal. Nucl. Chem. 304, 785-792.

Muller K., Nowak P.J., de Pan C., Marijnissen J.P., Paridaens D.A., Levendag P., Luyten G.P. (2005) Effectiveness of fractionated stereotactic radiotherapy for uveal melanoma, Int. J. Radiat. Oncol. Biol. Phys. 63, 116-122.

Nogueira P., Zankl M., Schlattl H., Vaz P. (2011) Dose conversion coefficients for monoenergetic electrons incident on a realistic human eye model with different lens cell populations, Phys. Med. Biol. 56, 6919-6934.

Pelowitz D. (2008) MCNPX User's Manual, Version 2.6.0. Los Alamos National Laboratory, Los Alamos, NM, USA. 
Righi S., Karaj E., Felici G., Di Martino F. (2013) Dosimetric characteristics of electron beams produced by two mobile accelerators, Novac7 and Liac, for intraoperative radiation therapy through Monte Carlo simulation, J. Appl. Clin. Med. Phys. 14, 1-17.

Rykers K., Uden G., Thompson V. (2003) Orbital lymphoma: a simple treatment using electrons, Med. Dosim. 28, 95-98.

Sakhaee M., Vejdani-Noghreiyan A., Ebrahimi-Khankook A. (2015) A comparison of simple and realistic eye models for calculation of fluence to dose conversion coefficients in a broad parallel beam incident of protons, Radiat. Phys. Chem. 106, 61-67.

Stannard C., Sauerwein W., Maree G., Lecuona K. (2013) Radiotherapy for ocular tumours, Eye 27, 119-127.

Swakon J., Olko P., Adamczyk D., Cywicka-Jakiel T., Dabrowska J., Dulny B., Grzanka L., Horwacik T., Kajdrowicz T., Michalec B., Nowak T. (2010) Facility for proton radiotherapy of eye cancer at IFJ PAN in Krakow, Radiat. Meas. 45, 1469-1471.
Taherkhani A., Mohammadi M., Saboori M.S., Changizi V. (2010) Evaluation of the physical characteristic of Cerrobend blocks used for radiation therapy, Int. J. Radiat. Res. 8, 93-101.

Terasawa T., Dvorak T., Ip S., Raman G., Lau J., Trikalinos T.A. (2009) Systematic review: charged-particle radiation therapy for cancer, Ann. Intern. Med. 151, 556-565.

Voke J. (1999) Radiation effects on the eye, part 1: infrared radiation effects on ocular tissue, Optom. Today 5, 22-28.

Weber D.C. et al. (2005) Proton beam radiotherapy versus fractionated stereotactic radiotherapy for uveal melanomas: A comparative study, Int. J. Radiat. Oncol. 63, 373-384.

Worgul B.V., Merriam G.R. Jr (1980) The lens epithelium and radiation cataracts: II. interphase death in the meridional rows? Radiat. Res. 84, 115-121.

Cite this article as: A. Vejdani-Noghreiyan, A. Ebrahimi-Khankook, E. Rahmani, M. Sakhaee. Dose calculation of different eye substructures using a realistic eye model when treating ocular tumors with electron therapy. Radioprotection 51(3), 179-186 (2016). 